\title{
Anatomy and Surgical Approach of the Ear and Temporal Bone
}

\author{
Brandon Isaacson ${ }^{1}[0$ \\ Received: 6 March 2018 / Accepted: 27 April 2018 / Published online: 1 August 2018 \\ (c) Springer Science+Business Media, LLC, part of Springer Nature 2018
}

\begin{abstract}
The temporal bone is one of the more complex structures at the skull base that houses the hearing and vestibular organs, numerous nerves, and vessels. A host of inflammatory and neoplastic processes can occur within the temporal bone that often necessitate permanent and frozen section pathologic examination. A number of simple to complex surgical procedures are used to manage temporal bone pathology. This chapter will provide a brief overview of normal temporal bone anatomy, common surgical approaches, normal histology, and indications for pathologic examination.
\end{abstract}

Keywords Temporal bone $\cdot$ Ear $\cdot$ Tympanoplasty $\cdot$ Mastoidectomy $\cdot$ Petrous bone $\cdot$ Paraganglioma $\cdot$ Skull base

\section{Introduction}

The temporal bone is a complex structure within the skull base that contains numerous critical structures including cranial nerves, vasculature, and the middle ear. A host of pathological conditions involve the temporal bone that can present with a myriad of symptoms. Understanding the complex anatomy and pathologic condition affecting the temporal bone is critical to establishing a differential diagnosis and management plan. This review will provide an overview of the anatomy of the temporal bone, common pathologic conditions, and the more common surgical approaches. The overview is summarized by a review of intraoperative scenarios leading to a request for intraoperative (frozen section) consultation with pathology.

\section{Anatomy}

The temporal bone is comprised of four distinct osseous segments including tympanic, mastoid, petrous, and squamous. The pinna attaches to the lateral surface of the temporal bone and opens up into the external auditory canal (EAC), which is an S-shaped tube that has a cartilaginous and osseous

Brandon Isaacson

Brandon.isaacson@utsouthwestern.edu

1 Department of Otolaryngology - HNS, UT - Southwestern Medical Center, 5323 Harry Hines Blvd., Dallas,

TX 75390-9035, USA component. The lateral third of the EAC is cartilaginous and is covered with thicker hair bearing skin (Fig. 1a, b), while the medial two-thirds is composed of the tympanic, squamous, and mastoid portions of the temporal bone. The fissures of Santorini are natural openings within the anterior cartilage in the lateral aspect of the canal that may allow infectious or neoplastic processes to extend from the parotid gland to the canal or the opposite scenario (Fig. 2a, b) [1] . The medial osseous EAC is lined with a very thin layer of stratified squamous epithelium without dermal elements (Fig. 3a, b). The tympanosquamous and tympanomastoid sutures are located within the osseous portion of the EAC and represent junction points between the respective osseous components of the temporal bone. These sutures can be very prominent and are sometimes relevant in various pathologic conditions including as a means of spread of an infectious or neoplastic process [2].

The tympanic membrane is attached to the medial end of the EAC by the annular ligament and separates the canal from the middle ear (Fig. 4). The tympanic membrane is comprised of three distinct layers from lateral to medial: stratified squamous epithelium, fibrous layer of lamina propria with radial and circumferential collagen bundles, and an inner most mucosal layer that is continuous with the middle ear epithelium (Fig. 5) [3]. The middle ear is a complex structure containing the auditory ossicles (malleus, incus, stapes), nerves (facial, chorda tympani, Jacobson's), ligaments and mucosal folds. The middle ear is also separated into different compartments (mesotympanum, epitympanum, hypotympanum, protympanum, and retrotympanum) [4]. 
Fig. 1 a Cross section of membranous (cartilaginous) external auditory canal (scanning magnification, H\&E stain). b Higher magnification of area within black box of a demonstrating stratified squamous epithelium with typical adnexal structures including a hair follicle (black arrow) $(\times 6$ magnification, $\mathrm{H} \& \mathrm{E}$ stain)
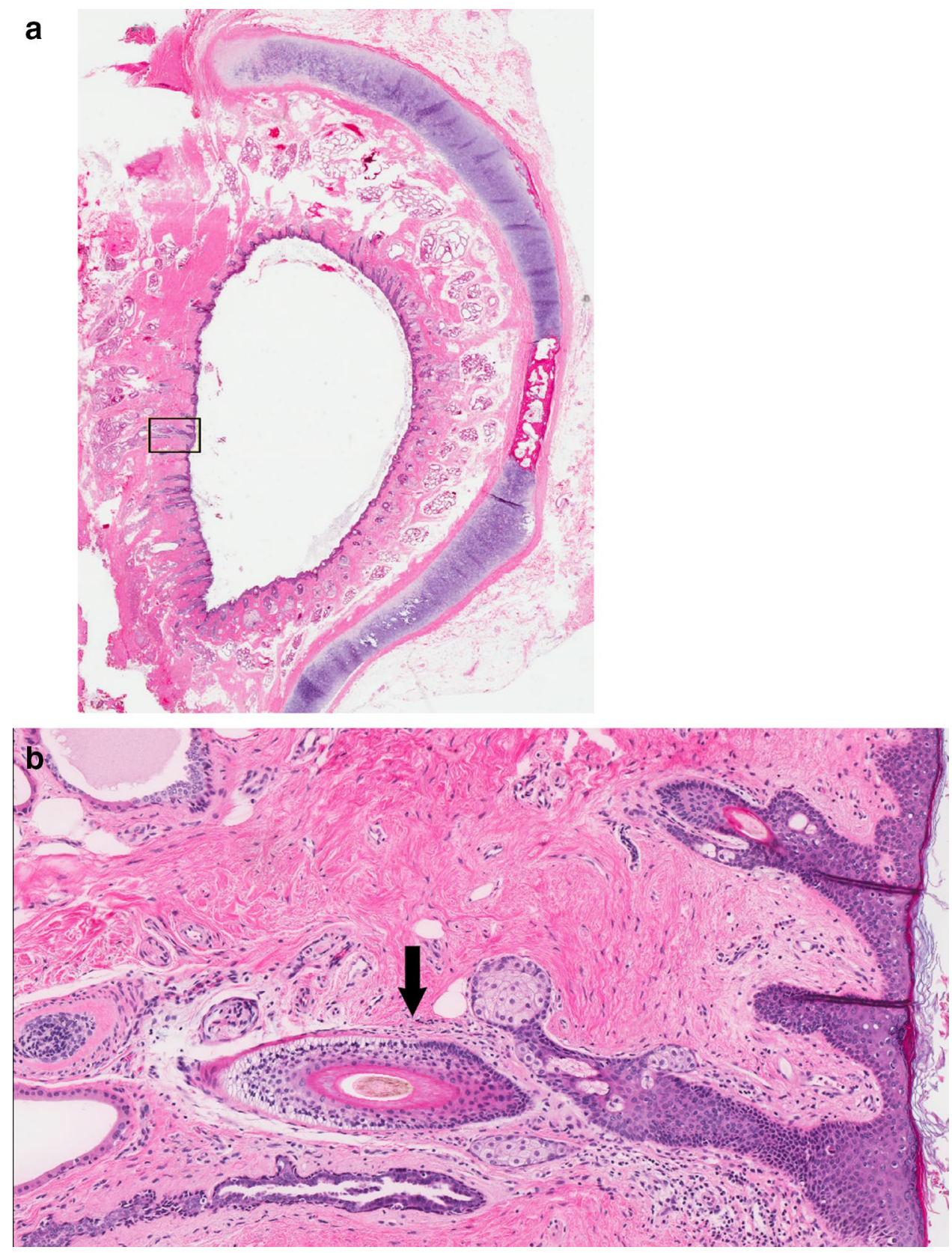

The epitympanum is defined as the space between the tegmen tympanum (roof of the middle ear) and the lateral process of the malleus with the lateral wall being comprised of the scutum and pars flaccida, and the medial wall being the bone covering the geniculate ganglion, and ampullated ends of the lateral and superior semicircular canals. The posterior aspect of the epitympanum connects to the mastoid air cell system via the aditus ad antrum. The incus body, and head and neck of the malleus are suspended in the central aspect of the epitympanum by the incudal ligament and the anterior and posterior malleolar ligaments respectfully. Several embryologically derived mucosal folds further segment the epitympanum into various compartments including
Prussak's space, and anterior epitympanic recess. The pathology of the mucosal folds often affects epitympanic and mastoid ventilation that is often the source of pars flaccida retraction and subsequent development of cholesteatoma [5].

The mesotympanum is circumferentially defined by the annulus and the lateral process of the malleus and contains the stapes superstructure, incus long process, malleus handle/umbo, tympanic facial nerve, oval and round windows. The oval and round windows are the only two openings between the middle and inner ear and are covered by the stapes footplate, and round window membrane respectively. The lateral boundary of the mesotympanum is the pars tensa of the tympanic membrane while the cochlear 
Fig. 2 a Longitudinal section of the membranous external auditory canal. Black box represents fissure of Santorini (scanning magnification, Hematoxylin and Eosin [H\&E] stain). b Higher magnification of fissure of Santorini enclosed in black box in $\mathbf{a}$ ( $\times 6$ magnification, H\&E stain)
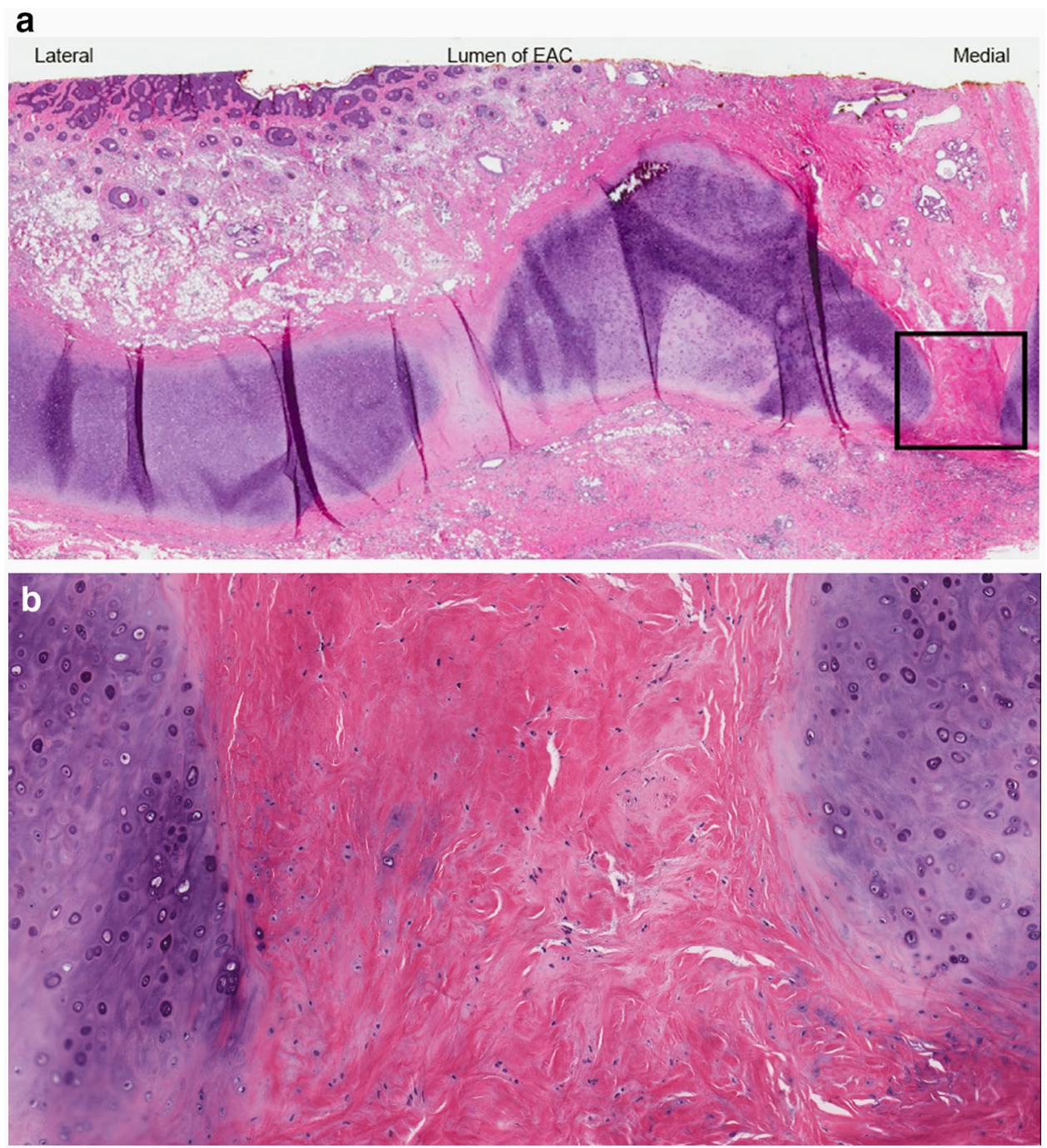

promontory serves as the medial boundary. The retrotympanum extends from the posterior annulus to the anterior border of the mastoid facial nerve and contains several spaces including the subpyrimidal space, sinus tympani, and sinus subtympanicum that often house residual cholesteatoma disease. The retrotympanum is separated from the hypotympanum by the funiculus, which is a bridge of bone that extends over the sub-cochlear cannliculus and contains Jacobson's nerve. The sub-cochlear canaliculus is a natural pathway medially that communicates with variable present air cells in inferior petrous apex [6]. The medial border of the hypotympanum is the bone covering the posteriorly located jugular bulb and the anteriorly located vertical petrous carotid artery. The superior border of the hypotympanum laterally is the inferior annulus. The protiniculum is a variable crest of bone the separates the hypotympanum from the protympanum [7]. The posterior border of the protympanum is the anterior annulus and contains the semicanal for the tensor tympani muscle, horizontal petrous carotid artery, osseous orifice of the eustachian tube [4] (Fig. 6).

The mastoid air cells system contains a large air cell known as the antrum, which communicates with the epitympanum via the aditus ad antrum. The antrum communicates with numerous smaller air cell systems in all directions. The posterior and superior osseous external auditory canal wall separates the mastoid from the EAC. The tegmen mastoideum, which is of variable thickness, is continuous with the tegmen tympani and serves as the roof of the mastoid. The mastoid tip and sigmoid sinus/posterior fossa plate serve as the inferior and posterior boundaries of the mastoid. The medial boundary of the mastoid is comprised of the semicircular canals, and vertical facial nerve. Variable pneumatization patterns occur within the temporal bone and a number of pathways allow for communication between the mastoid air cell system, middle ear, and the petrous apex [8].

The petrous segment is the most medial aspect of the temporal bone and contains the internal auditory canal, petrous 

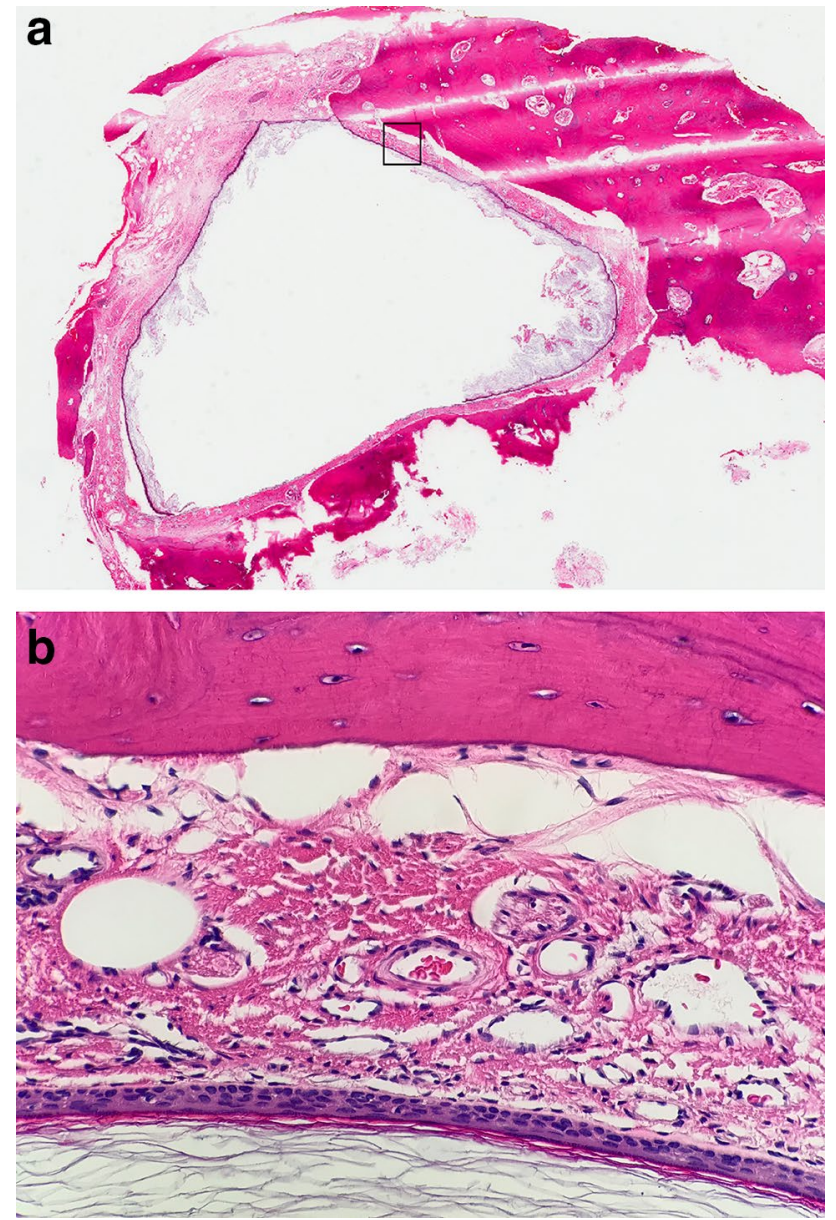

Fig. 3 a Cross section of osseous external auditory canal (scanning magnification, H\&E stain). b Higher magnification of the osseous canal within black box of a demonstrating stratified squamous epithelium and fibrovascular connective tissue lining bony EAC $(\times 8$ magnification, $\mathrm{H} \& \mathrm{E}$ stain)

carotid artery, jugular bulb, cochlea, vestibule, semicircular canals, vestibular aqueduct, endolymphatic sac, and intracanalicular, labyrinthine, and geniculate facial nerve. The 8th, 9th, 10th, and 11th cranial nerves extend into the temporal bone through the internal auditory canal and jugular foramen respectively. The petrous apex bone around the aforementioned structures can consist of air cells, bone marrow, or solid bone. The petrous apex is frequently divided into anterior and posterior segments by the internal auditory canal [8].

The anatomy of the temporal bone is quite complex and similarly the surgical approaches used to access the aforementioned areas range from simple to complex.

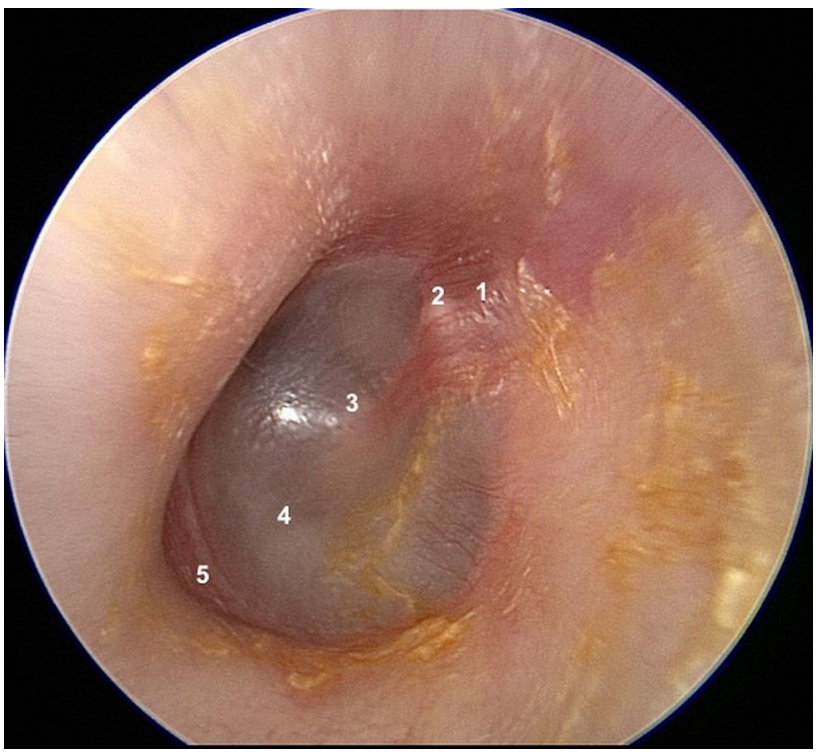

Fig. 4 An endoscopic view of a normal left tympanic membrane: 1 pars flaccida, 2 lateral process of malleus, 3 malleus umbo, 4 inferior central pars tensa, 5 fibrous annulus

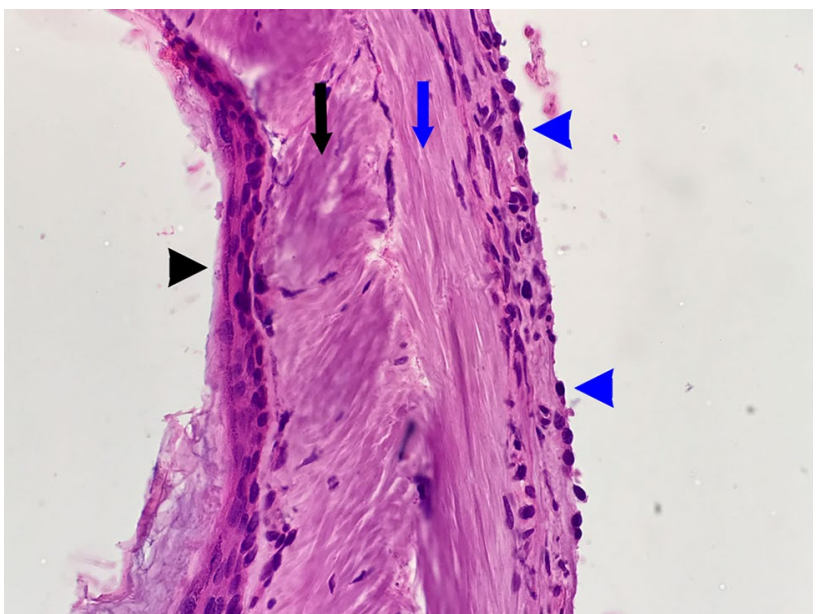

Fig. 5 Cross section through tympanic membrane. The black arrowhead demonstrates the outer or lateral surface of the tympanic membrane composed of stratified squamous epithelium. The blue arrowheads indicate the medial or middle ear surface of the tympanic membrane composed of cuboidal middle ear epithelium. The black arrow points towards the radial collagen fibers of the fibrous layer, while the blue arrow points towards the circular collagen fibers of the fibrous layer ( $\times 10$ magnification, $H \& E$ stain)

\section{Common Surgical Procedures}

Myringoplasty is a procedure typically used to repair a small tympanic membrane perforation, where the preexisting hole is used to deliver the graft material. The most 


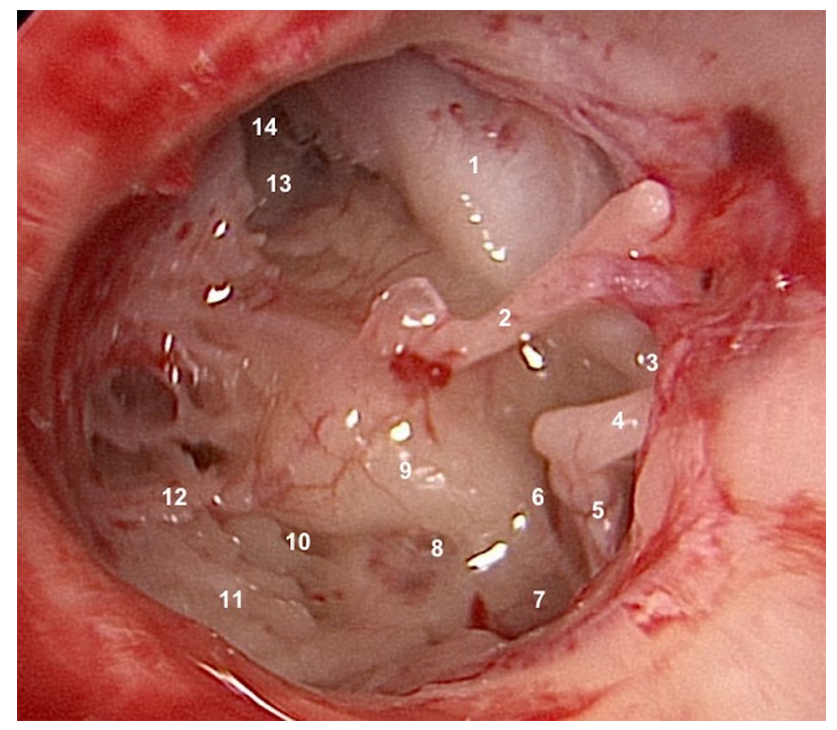

Fig. 6 An endoscopic view of the left middle ear after removal of the tympanic membrane: 1 semicanal of the tensor tympani muscle, 2 malleus handle, 3 tympanic facial nerve, 4 incus long process, 5 stapes capitulum and incudostapedial joint, 6 inferior margin of oval window niche, 7 sinus tympani, 8 round window niche, 9 cochlear promontory, 10 subcochlear canaliculus leading to inferior petrous apex, 11 high, non-dehiscent jugular bulb, 12 Jacobson's nerve, 13 horizontal petrous carotid artery, 14 eustachian tube

common etiologies for a tympanic membrane perforations include infection, trauma, and tympanostomy tube placement. Less common indications for myringoplasty include intratympanic cholesteatoma, granulation tissue, or rarely neoplasms. A myringoplasty is typically performed through the ear canal using either a surgical microscope or an endoscope. If a perforation is present the margins are excised to create a fresh wound and to ensure removal of any squamous epithelium that has migrated on the medial surface of the tympanic membrane. Removal of intratympanic cholesteatoma, granulation, or neoplasms typically results in a perforation that may be repaired using a myringoplasty technique. Potential indications for pathologic examination during myringoplasty include neoplasm or to assess for fungal elements. Intratympanic cholesteatoma is usually easily to identify on examination or at surgery. Typical autologous materials used during myringoplasty for repairing the tympanic membrane include fascia, fat, or cartilage. Processed cadaveric allografts and xenografts are also available for myringoplasty. Relative contraindications for myringoplasty include active middle ear infection, and eustachian tube dysfunction [9].

Tympanoplasty, unlike myringoplasty, entails elevation of a tympanomeatal flap to address pathology of the tympanic membrane, and/or the middle ear space. Perforation, cholesteatoma, ossicular chain pathology, and neoplasm are the most common indications for tympanoplasty.
Tympanoplasty surgery can be performed through the ear canal or via a postauricular incision using either a microscope or an endoscope for visualization. Similar to myringoplasty, the edges of a tympanic membrane perforation need to be excised. The surgery is typically performed by making incisions in the posterior ear canal skin adjacent to the tympanic membrane. The canal skin and tympanic membrane are elevated together, which allows the surgeon to visualize the entire middle ear space [10]. Fixation or erosion of the malleus, incus or stapes can be repaired using a tympanoplasty approach using either autologous material or prosthetic materials (PORP - partial ossicular replacement prosthesis, TORP — total ossicular replacement prosthesis). Cartilage is used to cover ossicular prosthetics, except for stapes implants, as these will typically extrude or become exposed through the tympanic membrane without coverage [11]. Explantation of middle ear prosthetics rarely if ever necessitates pathologic examination. Stapedectomy necessitates either creation of a fenestra in the footplate or partial/total removal [12]. Pathologic examination of the stapes superstructure is rarely indicated unless there is suspicion of a fibro-osseous pathology (Paget's, fibrous dysplasia, desmoplastic fibroma of bone).

Cholesteatoma typically originates in the middle ear either via tympanic membrane retraction, migration through a perforation, iatrogenic implantation, or rarely can be congenital. A tympanoplasty approach is typically used to remove cholesteatoma when confined to the middle ear, but a mastoidectomy becomes necessary with more extensive disease. A recent study by Kircher et al. examined the estimated yearly cost and utility of pathologic examination of cholesteatoma that amounted to over $\$ 1,000,000$. This same study of 178 cases showed no evidence of malignancy and concordance between surgeon's gross confirmation of cholesteatoma with pathologic examination was perfect [13]. A strong argument can also be made that pathological analysis of tissue should be performed as occasionally cholesteatoma can occur secondary to neoplastic obstruction of the external auditory canal or the eustachian tube [14].

Paraganglioma is the most common neoplasm found in the middle ear and typically originates from glomus bodies localized on Jacobson's nerve (glomus tympanicum) or the wall of the jugular bulb (glomus jugulare). Paragangliomas have a very characteristic appearance on exam and intraoperatively and specimens are typically sent as permanent sections to confirm the diagnosis [15]. Endolymphatic sac tumors, and hemangiomas may have a similar appearance on physical exam, but the presenting history, and preoperative imaging differs from paraganglioma in most cases [16]. Schwannomas or neuromas are occasionally identified within the middle ear and originate from the chorda tympani nerve, Jacobson's nerve, facial nerve, or rarely 8th nerve schwannomas may erode through the promontory 
and present in the middle ear [17]. The small volume of the middle ear space typically provides only a small volume of sample for pathologic examination. Frozen section analysis is occasionally indicated to confirm complete excision especially when managing facial nerve tumors as skip lesions may occur [18]. Frozen sections are sometimes used to determine if an adequate tissue sample is present for permanent pathologic examination or to differentiate an infectious from a neoplastic process.

Mastoidectomy is often performed in combination with tympanoplasty for chronic otitis media with and occasionally without cholesteatoma. Mastoidectomy after the initial post-auricular incision is the initial step for a number of otologic procedures including cochlear implantation, endolymphatic sac decompression, and for most of the presigmoid approaches to the lateral skull base. Mastoid surgery is often categorized as either being canal wall up or canal wall down referring to preservation or removal of the posterior-superior aspect of the osseous external auditory canal respectively. A cortical mastoidectomy, an intact canal wall technique, is typically performed in the setting of acute mastoiditis with subperiosteal abscess [19]. A surgical drill is used to remove the cortical bone covering the lateral mastoid air cells down to the antrum, which is the largest air cell connecting to the posterior aspect of the epitympanum via the aditus ad antrum. Removal of all of the lateral mastoid air cells covering the sigmoid sinus, tegmen, and posterior canal wall is consistent with a complete or canal wall up mastoidectomy and is typically performed for cholesteatoma and occasionally chronic otitis media without cholesteatoma. Canal wall up or complete mastoidectomy is also the initial step for most of the presigmoid skull base approaches. Canal wall down mastoidectomy requires the same steps as a complete mastoidectomy in addition to removal of the posterior superior osseous canal wall which exteriorizes the mastoid into the ear canal forming a single cavity. A modified radical mastoidectomy is a canal wall down technique where the tympanic membrane and ossicles are left in situ and the middle ear is left undisturbed. Modified radical mastoidectomy is most commonly performed for isolated epitympanic or attic cholesteatoma in an only hearing ear, or in patients with excellent hearing. A standard canal wall down mastoid tympanoplasty is similar to modified radical mastoidectomy except the tympanic membrane and the ossicular chain are repaired in the setting of a perforation, and ossicular discontinuity respectively. A radical mastoidectomy entails removal of the tympanic membrane and ossicles and plugging of the eustachian tube and is typically used for extensive cholesteatoma, or neoplasms. Extensive temporal bone paragangliomas, middle ear adenomas sometimes necessitate a radical or canal wall down mastoidectomy. Mastoid obliteration is sometimes referred to as subtotal petrosectomy where all of the air cells within the temporal bone are removed, the eustachian tube is plugged, the cavity is filled with a regional flap or free fat and the ear canal is closed. Indications for obliteration include intractable cerebrospinal fluid leak, end stage chronic ear disease, and extensive neoplasms that have resulted in profound hearing loss [20].

Typical specimens from mastoid surgery include diseased mucosa, cholesteatoma, paraganglioma, granulation tissue, and cholesterol granuloma. Rare pathology that can be encountered with these approaches include endolymphatic sac tumor, rhabdomyosarcoma, fibrous dysplasia, chondrosarcoma, chordoma, and desmoplastic fibroma of bone.

Intraoperative pathology consultation is occasionally required especially in the setting of neoplasms for margin control and in some infectious entities.

\section{Frozen Sections}

There are several scenarios where frozen section analysis of specimens facilitates surgical decision making. Cutaneous neoplasms involving the external auditory canal typically requires frozen section analysis to ensure adequate margin control during tumor excision. Obtaining tumor free surgical margins is one of the most important prognostic factors for successful excision of temporal bone cancers [21]. Basal cell cancer involving the pinna are best managed with Mohs surgery but if the tumor extends too medial into the membranous external auditory canal a lateral temporal bone resection is often necessary [22].

Frozen sections are sometimes ordered intraoperatively in order to establish that a representative tumor sample has been obtained in order to avoid a sampling error. Chondrosarcomas frequently have a gelatinous consistency and can be difficult to sample at the time of surgery as suctioning the tumor can aspirate the entire specimen [23]. Necrotizing otitis externa and skull base osteomyelitis are potentially life-threatening infections that mainly occur in diabetic or immunocompromised patients. Extensive skull base neoplasms can be difficult to distinguish from skull base osteomyelitis and surgical biopsy of the affected area is necessary to establish the diagnosis and to help direct antibiotic selection in the setting of an infection. Fungal stains and cultures should be requested in patients with skull base osteomyelitis especially in the setting of refractory or recurrent disease [24]. Frozen section analysis is sometimes obtained in patients with suspected paragangliomas as endolymphatic sac tumors, hemangiopericytoma, and metastatic renal cell carcinoma all grossly look similar and have an extensive vascular supply. 


\section{Conclusion}

Communication between the surgeon and pathologist is critical to making appropriate management recommendations in temporal bone pathology given the complex anatomy. A working understanding of the anatomy, surgical approaches, and pathologic entities affecting the temporal bone facilitates patient management. Frozen section analysis can be of critical importance depending on the clinical scenario.

\section{Compliance with Ethical Standards}

Conflict of interest Brandon Isaacson, MD, FACS declares that he has the following conflicts of interest. Advisory Boards: Med-el, Advanced Bionics. Consultant: Karl Storz Endoscopy, Olympus, Medtronic, Stryker.

Research Involving Human and Animal Rights This article does not contain any studies with human participants or animals performed by any of the authors.

\section{References}

1. Barrow HN, Levenson MJ. Necrotizing 'malignant' external otitis caused by Staphylococcus epidermidis. Arch Otolaryngol Head Neck Surg. 1992;118(1):94-6.

2. Kwong Y, Yu D, Shah J. Fracture mimics on temporal bone CT: a guide for the radiologist. Am J Roentgenol. 2012;199(2):428-34.

3. Lim DJ. Structure and function of the tympanic membrane: a review. Acta Otorhinolaryngol Belg. 1995;49(2):101-15.

4. Tarabichi M, Marchioni D, Presutti L, Nogueira JF, Pothier D. Endoscopic transcanal ear anatomy and dissection. Otolaryngol Clin N Am. 2013;46(2):131-54.

5. Marchioni D, Alicandri-Ciufelli M, Molteni G, Artioli FL, Genovese E, Presutti L. Selective epitympanic dysventilation syndrome. Laryngoscope 2010;120(5):1028-33.

6. Wick CC, Hansen AR, Kutz JW Jr, Isaacson B. Endoscopic infracochlear approach for drainage of petrous apex cholesterol granulomas: a case series. Otol Neurotol. 2017;38(6):876-81.

7. Jufas N, Marchioni D, Tarabichi M, Patel N. Endoscopic anatomy of the protympanum. Otolaryngol Clin $\mathrm{N}$ Am. 2016;49(5):1107-19.

8. Isaacson B, Kutz JW, Roland PS. Lesions of the petrous apex: diagnosis and management. Otolaryngol Clin N Am. 2007;40(3):479-519.
9. Anzola JF, Nogueira JF. Endoscopic techniques in tympanoplasty. Otolaryngol Clin N Am. 2016;49(5):1253-64.

10. Wick CC, Arnaoutakis D, Kaul VF, Isaacson B. Endoscopic lateral cartilage graft tympanoplasty. Otolaryngol Head Neck Surg. 2017;157(4):683-9.

11. Yawn RJ, Hunter JB, O'Connell BP, et al. Audiometric outcomes following endoscopic ossicular chain reconstruction. Otol Neurotol. 2017;38(9):1296-300.

12. Isaacson B, Hunter JB, Rivas A. Endoscopic stapes surgery. Otolaryngol Clin N Am. 2018;15:415-28.

13. Kircher ML, Thottam PJ, Bojrab DI, Babu SC. Utility and cost analysis of cholesteatoma histopathologic evaluation. Laryngoscope 2014;124(2):538-40.

14. Ferlito A. A review of the definition, terminology and pathology of aural cholesteatoma. J Laryngol Otol. 1993;107(6):483-8.

15. Killeen DE, Wick CC, Hunter JB, et al. Endoscopic management of middle ear paragangliomas: a case series. Otol Neurotol. 2017;38(3):408-15.

16. Wick CC, Manzoor NF, Semaan MT, Megerian CA. Endolymphatic sac tumors. Otolaryngol Clin N Am. 2015;48(2):317-30.

17. Sozen E, Ucal YO, Kabukcuoglu F, Celebi I, Dadas B. An incidental middle-ear mass: Jacobson's nerve schwannoma. Otol Neurotol. 2012;33(5):e37-8.

18. McRackan TR, Wilkinson EP, Rivas A. Primary tumors of the facial nerve. Otolaryngol Clin N Am. 2015;48(3):491-500.

19. Goycoolea MV. Mastoid and tympanomastoid procedures in otitis media: classic mastoidectomy (simple, modified, and radical) and current adaptations; open-cavity, closed-cavity, and intact-bridge tympanomastoidectomy. Otolaryngol Clin N Am. 1999;32(3):513-23.

20. Vercruysse JP, van Dinther JJ, De Foer B, et al. Long-term results of troublesome CWD cavity reconstruction by mastoid and epitympanic bony obliteration (CWR-BOT) in adults. Otol Neurotol. 2016;37(6):698-703.

21. Gidley PW. Managing malignancies of the external auditory canal. Expert Rev Anticancer Ther. 2009;9(9):1277-82.

22. Breen JT, Roberts DB, Gidley PW. Basal cell carcinoma of the temporal bone and external auditory canal. Laryngoscope 2017. https://doi.org/10.1002/lary.26785.

23. Carlson ML, O'Connell BP, Breen JT, et al. Petroclival chondrosarcoma: a multicenter review of 55 cases and new staging system. Otol Neurotol. 2016;37(7):940-50.

24. Blyth CC, Gomes L, Sorrell TC, da Cruz M, Sud A, Chen SC. Skull-base osteomyelitis: fungal vs. bacterial infection. Clin Microbiol Infect. 2011;17(2):306-11. 